\title{
Postoperative infusion of a low dose of dexmedetomidine reduces intravenous consumption of sufentanil in patient-controlled analgesia
}

\author{
Dae-eun Kweon, Youngbin Koo, Seonyi Lee, Kumhee Chung, Sowoon Ahn, \\ and Chunghyun Park
}

Department of Anesthesiology and Pain Medicine, CHA Bundang Medical Center, CHA University, Seongnam, Korea

\begin{abstract}
Background: Combining adjunctive medications with patient-controlled analgesia (PCA) has been used to minimize opioid related side-effects. The aim of this study was to evaluate whether postoperative infusion of a sub-sedative dose of dexmedetomidine can reduce opioid consumption and opioid related side-effects.

Methods: We selected 60 patients from 18 to 60 years old with an American Society of Anesthesiologists physical status of 1-2 who were scheduled for elective surgery. The types of surgery were limited to thoracoscopic wedge resection of the lung and pulmonary wedge resection under a mini-thoracotomy. Patients received PCA with sufentanil upon arrival in the recovery room, along with a separate continuous infusion of dexmedetomidine that was not mixed in the PCA but started at the same time. Patients were randomly allocated to two groups: dexmedetomidine $0.15 \mu \mathrm{g} / \mathrm{kg} / \mathrm{h}$ was administered to patients in group D and normal saline was administered to patients in group C. The visual analogue scale (VAS) pain score, blood pressure, pulse rate, and respiratory rate were measured at each assessment. PCA related side-effects were evaluated.

Results: The VAS pain score was significantly lower in the dexmedetomidine group. Patients in the dexmedetomidine group required significantly less PCA at postoperative 1-4, 4-8, and 8-24 h time intervals. The incidence of nausea was significantly less in the dexmedetomidine group, and levels of sedation and hemodynamic variables except for blood pressure at postoperative $8 \mathrm{~h}$ were similar between the groups.

Conclusions: In conclusion, a postoperatively administered sub-sedative dose of dexmedetomidine reduces PCA sufentanil consumption and decreases nausea.
\end{abstract}

Keywords: Dexmedetomidine; Patient-controlled analgesia; Sufentanil.

Corresponding author: Chunghyun Park, M.D., Ph.D.

Department of Anesthesiology and Pain Medicine, CHA Bundang Medical Center, CHA University, 82, Gumi-ro 173beon-gil, Bundang-gu, Seongnam 13620, Korea

Tel: 82-31-780-5431, Fax: 82-31-701-9433, Email: anesthpark@cha.ac.kr

ORCID: https://orcid.org/0000-0003-1916-6644

Received: March 14, 2017. Revised: May 31, 2017 (1st); June 14, 2017 (2nd). Accepted: June 14, 2017.

Korean J Anesthesiol 2018 June 71(3): 226-231

https://doi.org/10.4097/kja.d.18.27056

(c) This is an open-access article distributed under the terms of the Creative Commons Attribution Non-Commercial License (http://creativecommons.org/ licenses/by-nc/4.0/), which permits unrestricted non-commercial use, distribution, and reproduction in any medium, provided the original work is properly cited. 


\section{Introduction}

Postoperative pain can cause various complications, and active control is needed [1]. Patient-controlled analgesia (PCA) with an opioid is capable of satisfying the different analgesic ranges required for individual patients [2]. Opioids are widely used for PCA because of their excellent analgesic effect, but can cause various complications. Combining adjunctive medications, including antiemetics, non-steroidal anti-inflammatory drugs (NSAIDs), and ketamine, has been used to minimize opioid related side-effects $[3,4]$.

Dexmedetomidine, a highly selective $\alpha_{2}$-adrenoreceptor agonist, has sedative, anxiolytic, and analgesic effects. Unlike opioids, it does not cause respiratory depression [5]. Recent applications of dexmedetomidine include its use as an adjunct to general anesthesia to reduce the need for anesthetics for maintenance and postoperative analgesics [6]. For this reason, there has been an attempt to investigate whether the use of dexmedetomidine for PCA is effective in reducing the use of opioids, thereby reducing opioid related side-effects $[7,8]$. In previous studies, however, dexmedetomidine was mixed with PCA drugs, leading to variations in the dosage of dexmedetomidine used according to the severity of a patient's pain level. In this study, a constant amount of dexmedetomidine was administered separately from the PCA by an infusion pump.

In our study, the main hypothesis was that administration of dexmedetomidine after thoracic surgery would reduce the use of sufentanil in a prospective, randomized and double-blind study design. We also evaluated the efficacy of a sub-sedative dose of dexmedetomidine after surgery to reduce pain intensity and adverse effects of PCA.

\section{Materials and Methods}

\section{Enrollment}

The study was approved by the Institutional Review Board and registered with the Clinical Research Information Service RCT number. After obtaining written informed consent, we enrolled 60 patients from 18 to 60 years old with an American Society of Anesthesiologists physical status of I-II who were scheduled to undergo elective thoracic surgery. The types of surgery were limited to thoracoscopic wedge resection and minimally invasive pulmonary wedge resection under a mini-thoracotomy. Patients with a history of neurological and psychological deficits, hepato-renal dysfunctions, heart disease, long-term use of analgesic or sedative drugs, alcoholism or drug addiction, known sensitivity to any of the medications used, and those with a history of severe nausea and vomiting after intravenous (IV) PCA were excluded from the study. The study was a prospective, double-blind, and patients were randomly assigned to receive dexmedetomidine (group D) or to the control group (group C).

\section{Intraoperative management}

Premedication was not given to all patients. After arrival in the operating room, general anesthesia was induced with propofol and atracurium and tracheal intubation was performed. Anesthesia was maintained with $\mathrm{O}_{2}$-air-desflurane. After arrival in the recovery room, both groups received an IV PCA pump (AutoMed $3000^{\circledR}$, Ace Medical, Korea) with $100 \mathrm{ml}$ of normal saline mixed with $150 \mu \mathrm{g}$ of sufentanil. The basal rate was set to $2 \mathrm{ml} /$ $\mathrm{h}$, the bolus dose was $0.5 \mathrm{ml}$, and the lockout interval was set to 15 minutes. At the same time, the experimental drug was administered separately from the PCA by an infusion pump. The total dose of dexmedetomidine (Precedex ${ }^{\circledR}$, Hospira, USA) was calculated $(0.15 \mu \mathrm{g} \times$ weight $\times 24 \mathrm{~h})$ and mixed in normal saline to obtain a total volume of $120 \mathrm{ml}$. Infusion was maintained for $24 \mathrm{~h}$ at a rate of $5 \mathrm{ml} / \mathrm{h}$. The placebo was made with normal saline using the same volume. As a result, group $\mathrm{D}$ received dexmedetomidine at a dose of $0.15 \mu \mathrm{g} / \mathrm{kg} / \mathrm{h}$ for $24 \mathrm{~h}$, and group C received the same volume of saline.

The visual analogue scale (VAS) pain score, blood pressure, and pulse rate were measured every 15 minutes in the recovery room. The VAS was measured at resting and at motion. When the VAS at resting state was 4 or more, $0.01 \mu \mathrm{g} / \mathrm{kg}$ of sufentanil was administered as a rescue drug. To titrate the exact dose of sufentanil, a very small amount of rescue sufentanil was administered several times based on patient need. After the VAS at resting state decreased to 3 or less, the patient was transferred to the ward. At $4 \mathrm{~h}, 8 \mathrm{~h}$, and $24 \mathrm{~h}$ postoperatively, the patients were reevaluated based on the VAS pain score (resting and motion) and the amount of sufentanil consumed by time interval (first postoperative $1 \mathrm{~h}, 1-4 \mathrm{~h}, 4-8 \mathrm{~h}$, and $8-24 \mathrm{~h}$ ), and the severity of side effects, heart rate, and blood pressure were measured at $1 \mathrm{~h}$, $4 \mathrm{~h}, 8 \mathrm{~h}$, and $24 \mathrm{~h}$.

We assessed side-effects such as nausea and vomiting (0: no symptoms, 1: occurring once, 2: occurring more than 2 times but tolerable, 3 : severe enough to require medication), pruritus (0: no symptoms, 1: partial pruritus, 2: systemic pruritus but tolerable, 3: severe enough to require medication), and sedation ( 0 : no sedation at all, 1: eyes open when calling name, 2: eyes open when weakly shaken, 3: eyes open with a strong stimulus) with a scoring scale. If severe nausea and vomiting occurred, $4 \mathrm{mg}$ of ondansetron was administered intravenously as a rescue drug.

\section{Sample size}

The sample size was calculated using $\mathrm{G}^{*}$ power 3.1.9 (Franz Faul, Kiel University, Germany). Power analysis to determine 
the sample size used a primary endpoint defined as sufentanil consumption at $24 \mathrm{~h}$. This sufentanil consumption was based on a prior study [8]. In the study, the mean \pm SD amount of morphine consumption over $24 \mathrm{~h}$ with and without dexmedetomidine was $23.3 \pm 10$ and $32.8 \pm 12.4 \mathrm{mg}$, respectively, indicating a $29 \%$ reduction in morphine use with dexmedetomidine. Using a power of $80 \%$, level of statistical significance of $\alpha=0.05, \beta=0.2$, and calculated effect size of 0.843 , a sample size of 24 patients per group was required. Considering a dropout rate of $20 \%$, we assigned 30 patients to each group.

\section{Statistical methods}

Statistical analyses were conducted using SPSS 13.0 (USA). Student's t-tests for continuous variables and Fischer's exact tests for categorical variables were used to compare demographic characteristics and surgical data between the groups. The data distribution was evaluated using the Kolmogorov-Smirnov test. The VAS pain scores, sufentanil consumption, and hemodynamic parameters were analyzed by the Student's t-test. Differences in the severity of adverse events were analyzed by Fisher's exact tests. All results are expressed as the mean \pm SD. All statistics were two-tailed, and $\mathrm{P}$ values $<0.05$ were considered significant.

\section{Results}

Thirty patients were assigned to each group, although two patients in group D were excluded from the experiment (Fig. 1). There were no significant differences in age, height, weight, and duration of anesthesia between the two groups (Table 1).

The VAS pain score, which is an indicator of the analgesic effect, was significantly lower in group D than in group $C$ at rest and motion (Fig. 2). There was no statistically significant difference in the PCA drug consumption between the two groups during the first $1 \mathrm{~h}$ postoperatively. However, there were significant differences in the amount of sufentanil consumed for each time interval (1-4 h, 4-8 h, 8-24 h, and the total $24 \mathrm{~h}$ cumulative consumption) between the groups (Fig. 3). There was no significant differences in the incidence of vomiting, pruritus, and level of sedation between the two groups, although the incidence of nausea in group $\mathrm{D}$ was significantly lower than in group $\mathrm{C}(\mathrm{P}=0.021$, Table 2$)$. There was no significant difference in heart rate between the two groups at all time points (Fig. 4A). Mean blood pressure was significantly lower in group $\mathrm{D}$ than in group $\mathrm{C}$ at $8 \mathrm{~h}$ postoperatively $(\mathrm{P}=0.007)$, but there was no significant difference at the other time points (Fig. 4B).

\section{Discussion}

Postoperative pain requires aggressive management because it can cause side-effects such as adverse cardiovascular effects, respiratory depression, digestive and urinary dysfunction, and neuroendocrine dysfunction, as well as suffering from the pain itself [1]. PCA minimizes pharmacokinetic and pharmacodynamic differences between patients by allowing patients to

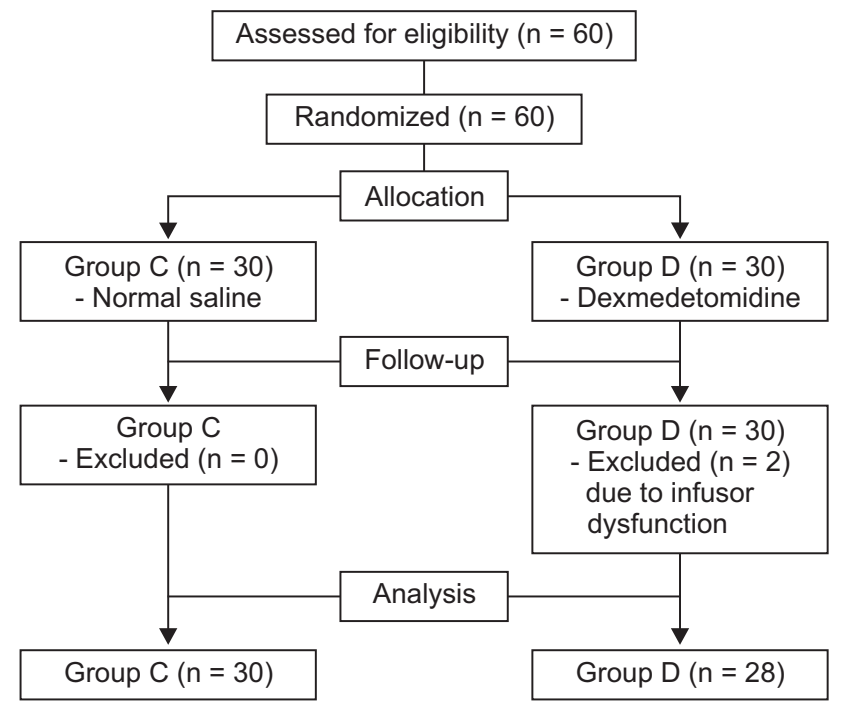

Fig. 1. Patient enrollment and CONSORT flow chart.

Table 1. Demographic Data of Patients

\begin{tabular}{lccc}
\hline & $\begin{array}{c}\text { Group C } \\
(\mathrm{n}=30)\end{array}$ & $\begin{array}{c}\text { Group D } \\
(\mathrm{n}=28)\end{array}$ & P value \\
\hline Age $(\mathrm{yr})$ & $39.8 \pm 17.7$ & $37.9 \pm 17.4$ & 0.699 \\
Gender (M/F) & $21 / 9$ & $18 / 10$ & 0.950 \\
Body weight $(\mathrm{kg})$ & $63.9 \pm 10.7$ & $60.5 \pm 8.2$ & 0.193 \\
Height $(\mathrm{cm})$ & $166.2 \pm 11.1$ & $166.3 \pm 11.1$ & 0.987 \\
Type of sugery & & & 0.271 \\
$\quad$ Thoracoscopic wedge resection & 24 & 5 & 0.368 \\
$\quad$ Mini-thoracotomy wedge resection & 6 & $39.3 \pm 6.8$ & \\
Duration of anesthesia (min) & $38.5 \pm 7.7$ & & \\
\hline
\end{tabular}

All measured values are presented as mean \pm SD or number of patients. 
A

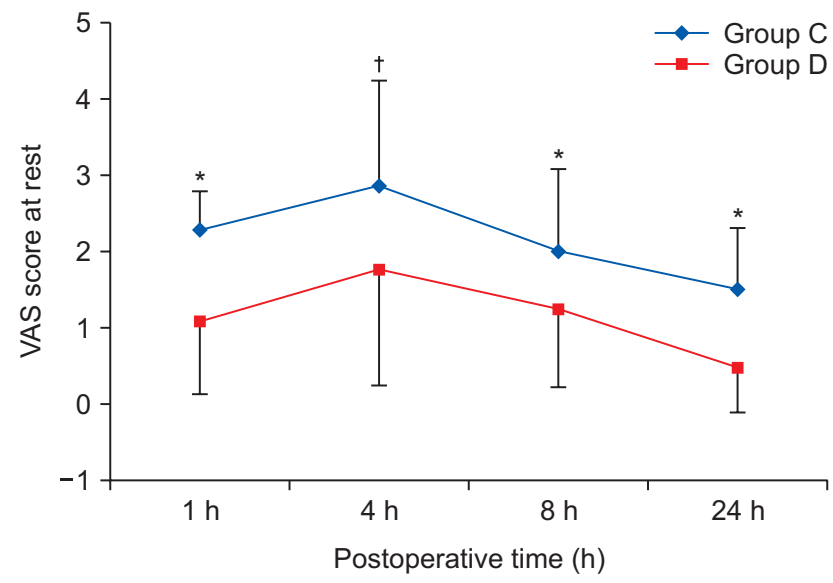

B

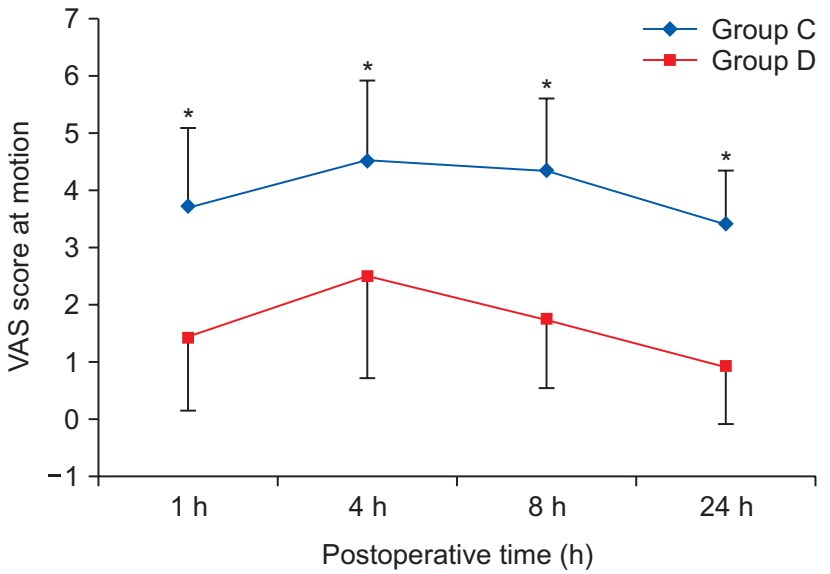

Fig. 2. Postoperative visual analogue scale (VAS) score at rest (A) and at motion (B). ${ }^{*, \dagger}$ Indicates that there was significantly lower in group D than in group $\mathrm{C}\left({ }^{*} \mathrm{P}<0.001,{ }^{\dagger} \mathrm{P}=0.007\right)$. All measured values are presented as mean $\pm \mathrm{SD}$.

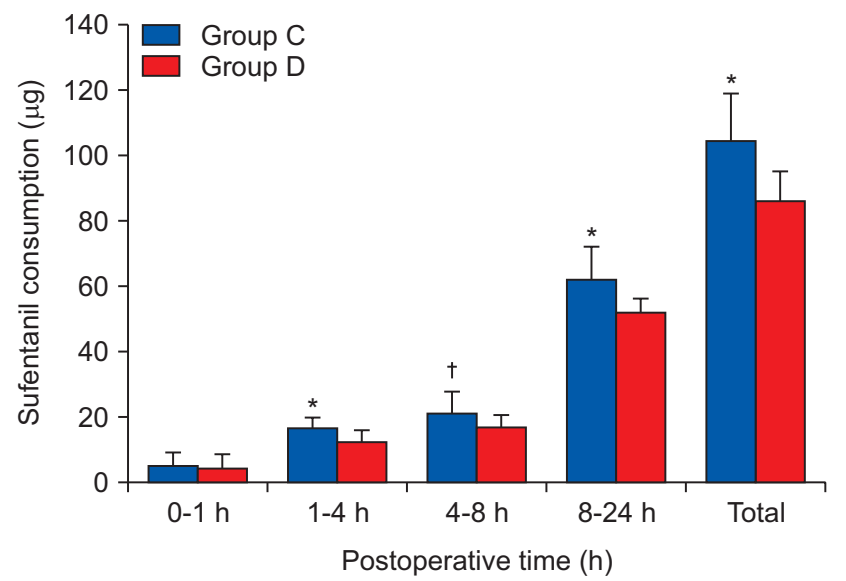

Fig. 3. The amount of postoperative sufentanil consumption. All measured values are presented as mean \pm SD. ${ }^{*,+}$ Indicate significant differences in using dose of sufentanil between the groups $\left({ }^{*} \mathrm{P}<0.001\right.$, $\left.{ }^{\dagger} \mathrm{P}=0.008\right)$.

adjust the amount of medication themselves. Additionally, PCA has the advantage of reducing a patient's anxiety due to delayed effects from intermittent administration, reducing side effects, and shortening the length of hospital stays $[9,10]$.

Opioids are widely used for PCA because of their excellent analgesic effect, but can cause nausea, vomiting, pruritus, and respiratory depression [11]. These side-effects may cause the patient to stop PCA, leading to insufficient pain management. A multimodal approach is used to maximize pain relief and minimize opioid-related side-effects. To prevent complications such as nausea and vomiting, the addition of antiemetics or reducing the consumption of opioids by adding NSAIDs or ketamine can be used [3,4].

Dexmedetomidine, a drug that binds 8 times more selec-
Table 2. Adverse Effects during PCA

\begin{tabular}{lcccccc}
\hline \multirow{2}{*}{ Effects } & Group & \multicolumn{5}{c}{ Score } \\
\cline { 3 - 6 } & & 0 & 1 & 2 & 3 & P value \\
\hline \multirow{2}{*}{ Nausea } & $\mathrm{C}(\mathrm{n}=30)$ & 17 & 2 & 3 & 8 & $0.021^{*}$ \\
& $\mathrm{D}(\mathrm{n}=28)$ & 22 & 5 & 0 & 1 & \\
Vomiting & $\mathrm{C}(\mathrm{n}=30)$ & 26 & 1 & 0 & 3 & 1.000 \\
& $\mathrm{D}(\mathrm{n}=28)$ & 25 & 1 & 0 & 2 & \\
Seduritus & $\mathrm{C}(\mathrm{n}=30)$ & 23 & 3 & 2 & 2 & 0.290 \\
& $\mathrm{D}(\mathrm{n}=28)$ & 26 & 2 & 0 & 0 & \\
& $\mathrm{C}(\mathrm{n}=30)$ & 23 & 7 & 0 & 0 & 0.432 \\
& $\mathrm{D}(\mathrm{n}=28)$ & 20 & 7 & 1 & 0 & \\
\hline
\end{tabular}

All measured values are number of patients.

tively to the $\alpha_{2}$ receptor than clonidine, has sedative, analgesic, and anxiolytic effects [12]. It is used in a variety of areas such as monitored anesthesia care, sedation in intensive care units, awake fiberoptic intubation, cardiac surgery, and neurosurgery. It is rarely used alone for analgesic effects and mainly used for sedation [5]. However, there is a decrease in blood pressure and heart rate in proportion to the dose used when the recommended dose for sedation is administered [6]. Therefore, when dexmedetomidine is used for analgesic purposes as in this study, it is important to observe whether unnecessary sedation or cardiovascular side effects occur.

Lin et al. [8] reported that combining dexmedetomidine and morphine for intravenous PCA enhanced analgesic effects, reduced the consumption of morphine, and reduced side-effects such as nausea. Lee et al. [7] also reported that the combination of dexmedetomidine and sufentanil for intravenous PCA showed better postoperative analgesic effects compared to the ketamine combined group. However, since dexmedetomidine 

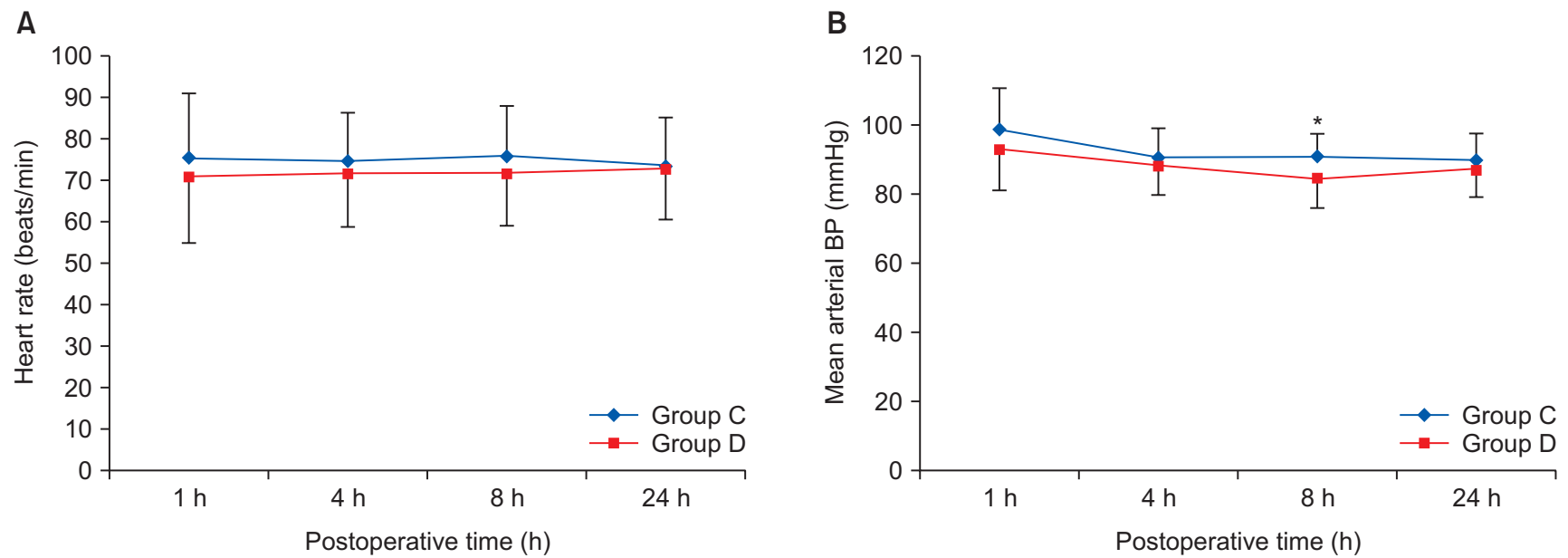

Fig. 4. Postoperative hemodynamic variables; (A) Heart rate and (B) Mean arterial blood pressure. All measured values are presented as mean \pm SD.

was infused as a mixture with morphine or sufentanil in those studies, the total amount of dexmedetomidine administered may have been different depending on the patient's needs. If a patient used a larger amount of PCA, more dexmedetomidine was administered simultaneously. This made it difficult to estimate the exact concentration of dexmedetomidine administered which is important because it may induce sedation in patients and to determine the adequate dose of dexmedetomidine as an adjunct drug for postoperative pain control. In this study, we tried to prevent using excessive doses of dexmedetomidine by adjusting the dose of dexmedetomidine per unit weight and hour. We also attempted to more clearly determine if the amount of opioid consumed was reduced with the addition of dexmedetomidine by using a very small amount of sufentanil at a time.

The $\alpha_{2}$ receptor agonists activate potassium channels to induce hyperpolarization of the cell membrane, which inhibits the action potential of excitatory cells in the central nervous system. In addition, suppression of calcium conduction into cells inhibits the release of excitatory neurotransmitters, resulting in analgesia [13]. It has been reported that the substantiagelatinosa of the dorsal horn of the spinal cord is the major site of analgesic action of $\alpha_{2}$ adrenoceptor agonists. For this reason, epidural or intrathecal administration have been considered preferable to intravenous administration for obtaining an analgesic effect [12].

In this study, dexmedetomidine was administered at a rate of $0.15 \mu \mathrm{g} / \mathrm{kg} / \mathrm{h}$, which is less than the maintenance dose of dexmedetomidine $(0.2-0.9 \mu \mathrm{g} / \mathrm{kg} / \mathrm{h})$ usually used in monitored anesthesia care [14]. In addition, since we did not give loading doses, it could be expected that the difference in the level of sedation between the two groups would be minimal. Ryu suggested that analgesia after intravenous administration of dexmedetomidine is due to the effect of sedation [5]. However, in this study, the analgesic effect was higher in the dexmedetomidine group, although there was no significant difference in the level of sedation between the two groups. According to Lee et al's report [7], $500 \mu \mathrm{g}$ of dexmedetomidine was mixed in $100 \mathrm{ml}$ of PCA which was infused at a basal rate of $2 \mathrm{ml} / \mathrm{h}$, indicating that more than $10 \mu \mathrm{g}$ of dexmedetomidine was infused in $1 \mathrm{~h}$. We could not determine the exact dose of dexmedetomidine used from that report because it did not mention the average weight of the patients and the total infused volume of PCA, and only the sparing ratio of PCA volume was reported [7]. Lin et al. mixed 100 mg of morphine $(1 \mathrm{mg} / \mathrm{ml})$ plus $500 \mu \mathrm{g}$ of dexmedetomidine $(5$ $\mu \mathrm{g} / \mathrm{ml}$ ) in normal saline to make a final PCA volume of $100 \mathrm{ml}$. During the $0-24 \mathrm{~h}$ postoperative period, the cumulative volume of PCA used was $23.3 \mathrm{ml}$. The average amount of dexmedetomidine used in the first $24 \mathrm{~h}$ was $116.5 \mu \mathrm{g}$ [8]. However, more than half of the cumulative volume was administered within the postoperative $0-4 \mathrm{~h}$ time interval, so it is ratiocinated that more than $58 \mu \mathrm{g}$ of dexmedetomidine was infused at a rate of approximately $0.25 \mu \mathrm{g} / \mathrm{kg} / \mathrm{h}$. Although none of the studies mentioned above reported that patients were sedated, a further study should be performed to determine the proper concentration of dexmedetomidine.

We compared the two groups based on the dose of sufentanil used in each time period rather than by the cumulative dose of sufentanil. Most PCA studies show a cumulative dose comparison because of the long duration of morphine. However, this comparison may blunt the results because if a single time interval has a large difference, it may affect the other time intervals based on the cumulative results.

Nausea is a side effect that can occur in patients receiving opioids, and is a common cause of the cessation of using PCA [15]. In this study, the incidence and severity of nausea decreased in the dexmedetomidine-administered group, which is probably due to the decrease in consumption of the PCA drug. 
The cardiovascular response of dexmedetomidine occurs through two mechanisms. Administering loading doses of dexmedetomidine stimulates $\alpha_{2} b$ receptors in vascular smooth muscle, resulting in an initial increase in blood pressure and a reflex drop in heart rate. The rise in blood pressure can be attenuated by avoiding bolus administration of the drug. After the first response which lasts 5 to 10 minutes, the blood pressure decreases due to central sympathetic dystrophy. Presynaptic $\alpha_{2}$ receptors are also stimulated, thereby decreasing norepinephrine release, which causes a drop in blood pressure and heart rate [16]. In this study, there was no clinically significant difference in blood pressure and heart rate between the two groups. This may have been due to the lack of administration of a loading dose and an infusion rate that was lower than the rate used for sedative purposes.
The results of this study showed that administration of dexmedetomidine together with postoperative PCA exerted a high analgesic effect and reduced drug consumption. The degree of sedation and hemodynamic changes did not differ significantly between the two groups, which implies that dexmedetomidine can be used safely in combination with PCA. Importantly, the frequency and severity of nausea also decreased, which could possibly lead to an increase in patient satisfaction with this method for postoperative pain control.

\section{ORCID}

Sowoon Ahn, https://orcid.org/0000-0002-2990-528X

Chunghyun Park, https://orcid.org/0000-0003-1916-6644

\section{References}

1. Hopf HW, Weitz S. Postoperative pain management. Arch Surg 1994; 129: 128-32.

2. Song SO, Jee DL, Koo Bu. Analysis of 1,590 patients of IV-PCA for postoperative pain management. J Korean Pain Soc 1996; 9: 354-62.

3. Lin TF, Yeh YC, Yen YH, Wang YP, Lin CJ, Sun WZ. Antiemetic and analgesic-sparing effects of diphenhydramine added to morphine intravenous patient-controlled analgesia. Br J Anaesth 2005; 94: 835-9.

4. Michelet P, Guervilly C, Hélaine A, Avaro JP, Blayac D, Gaillat F, et al. Adding ketamine to morphine for patient-controlled analgesia after thoracic surgery: influence on morphine consumption, respiratory function, and nocturnal desaturation. Br J Anaesth 2007; 99: 396-403.

5. Ryu J. Clinical applications of alpha2 adrenoceptor agonist. Anesth Pain Med 2010; 5: 177-82.

6. Carollo DS, Nossaman BD, Ramadhyani U. Dexmedetomidine: a review of clinical applications. Curr Opin Anaesthesiol 2008; 21 : 457-61.

7. Lee W, Shin JD, Choe K, Kim MH. Comparison of dexmedetomidine and ketamine for the analgesic effect using intravenous patientcontrolled analgesia after gynecological abdominal surgery. Korean J Anesthesiol 2013; 65(6 Suppl): S132-4.

8. Lin TF, Yeh YC, Lin FS, Wang YP, Lin CJ, Sun WZ, et al. Effect of combining dexmedetomidine and morphine for intravenous patientcontrolled analgesia. Br J Anaesth 2009; 102: 117-22.

9. Bennett RL, Batenhorst RL, Bivins BA, Bell RM, Graves DA, Foster TS, et al. Patient-controlled analgesia: a new concept of postoperative pain relief. Ann Surg 1982; 195: 700-5.

10. Wasylak TJ, Abbott FV, English MJ, Jeans ME. Reduction of postoperative morbidity following patient-controlled morphine. Can J Anaesth 1990; 37: 726-31.

11. Dahan A, Aarts L, Smith TW. Incidence, reversal, and prevention of opioid-induced respiratorydepression. Anesthesiology 2010; 112: 22638 .

12. Khan ZP, Ferguson CN, Jones RM. Alpha-2 and imidazoline receptor agonists. Their pharmacology and therapeutic role. Anaesthesia 1999; 54: 146-65.

13. Gertler R, Brown HC, Mitchell DH, Silvius EN. Dexmedetomidine: a novel sedative-analgesic agent. Proc (Bayl Univ Med Cent) 2001; 14: 13-21.

14. Park JH, Kwon JY. Remifentanil or dexmedetomidine for monitored anesthesia care during cataract surgery under topical anesthesia. Korean J Anesthesiol 2012; 63: 92-3.

15. Woodhouse A, Mather LE. Nausea and vomiting in the postoperative patient-controlled analgesia environment. Anaesthesia 1997; 52: 770-5.

16. Afonso J, Reis F. Dexmedetomidine: current role in anesthesia and intensive care. Rev Bras Anestesiol 2012; 62: 118-33. 